\title{
The Importance of Highly Connected Building Units in Reticular Chemistry: Thoughtful Design of Metal-Organic Frameworks
}

\author{
Vincent Guillerm ${ }^{\dagger}$ and Mohamed Eddaoudi*†
}

${ }^{\dagger}$ King Abdullah University of Science and Technology (KAUST), Division of Physical Sciences and Engineering, Advanced Membranes \& Porous Materials Center (AMPM), Functional Materials Design, Discovery \& Development Research Group (FMD3), Thuwal 23955-6900, Kingdom of Saudi Arabia. *Corresponding author: mohamed.eddaoudi@kaust.edu.sa

\begin{tabular}{|c|c|c|c|c|c|}
\hline \multirow[b]{2}{*}{ Date $^{a}$} & \multicolumn{2}{|c|}{ Connectivity } & \multirow[b]{2}{*}{ vertex figure $^{c}$} & \multirow[b]{2}{*}{ Metal(s) } & \multirow[b]{2}{*}{ Access the related work } \\
\hline & MBB & SBB & & & \\
\hline 8/20/1997 & 4 & & $4 \mathrm{MR}$ & $\mathrm{Cu}$ & https://doi.org/10.1246/cl.1997.1219 \\
\hline 9/17/1999 & 6 & & oct & $\mathrm{Zn}$ & https://doi.org/10.1038/46248 \\
\hline $1 / 18 / 2001$ & 6 & & oct & $\mathrm{Cu}$ & https://doi.org/10.1246/cl.2001.332 \\
\hline $11 / 6 / 2001$ & 10 & & pap & $\mathrm{Zn}$ & https://doi.org/10.1039/B108684G \\
\hline $11 / 6 / 2001$ & 12 & & cuo & $\mathrm{Zn}$ & https://doi.org/10.1039/B108684G \\
\hline $3 / 12 / 2004$ & 6 & & tpr & $\mathrm{Cr}$ & https://doi.org/10.1002/anie.200454250 \\
\hline $10 / 5 / 2004$ & 4 & & $4 \mathrm{MR}$ & Co & https://doi.org/10.1021/ic048612y \\
\hline $10 / 5 / 2004$ & 4 & & tet & Co & https://doi.org/10.1021/ic048612y \\
\hline $10 / 5 / 2004$ & & 12 & cuo & Co & https://doi.org/10.1021/ic048612y \\
\hline $4 / 26 / 2005$ & 8 & & trans-bicapped octahedron & $\mathrm{Zn} / \mathrm{Co}$ & https://doi.org/10.1002/adma.200500867 \\
\hline $10 / 28 / 2005$ & 8 & & hourglass & Co & https://doi.org/10.1002/chem.200501340 \\
\hline $12 / 4 / 2005$ & 3 & & $3 \mathrm{MR}$ & $\mathrm{Zn}$ & https://doi.org/10.1002/anie.200504312 \\
\hline $3 / 8 / 2006$ & 6 & & $6 \mathrm{MR}$ & $\mathrm{Zn} / \mathrm{Mn}$ & https://doi.org/10.1246/cl.2006.526 \\
\hline $5 / 20 / 2006$ & 8 & & cub & Co & https://doi.org/10.1021/ja063538z \\
\hline $8 / 5 / 2006$ & 8 & & cub & $\mathrm{Mn}$ & https://doi.org/10.1021/ja0656853 \\
\hline $7 / 19 / 2007$ & 6 & & tpr & $\mathrm{Cu}$ & https://doi.org/10.1021/ja0753952 \\
\hline $10 / 5 / 2007$ & 5 & & spr & $\mathrm{Zn}$ & https://doi.org/10.1021/ja0776778 \\
\hline $10 / 5 / 2007$ & & 12 & cuo & $\mathrm{Zn}$ & $\begin{array}{l}\text { https://doi.org/10.1021/ja0776778 } \\
\text { https://doi.org/10.1021/ja710123sS0002- }\end{array}$ \\
\hline $11 / 7 / 2007$ & & 24 & rco & $\mathrm{Cu}$ & $\begin{array}{l}\text { 7863(71)00123-9 } \\
\text { https://doi.org/10.1021/ja710123sS0002- }\end{array}$ \\
\hline $11 / 7 / 2007$ & 3 & & $3 \mathrm{MR}$ & $\mathrm{Cu}$ & $7863(71) 00123-9$ \\
\hline $7 / 24 / 2008$ & 12 & & cuo & $\mathrm{Zr}$ & https://doi.org/10.1021/ja8057953 \\
\hline $3 / 3 / 2009$ & 12 & & cuo & $\mathrm{Al}$ & https://doi.org/10.1002/anie.200901409 \\
\hline 3/5/2009 & & 6 & oct & $\mathrm{Cu}$ & https://doi.org/10.1021/ja901731z \\
\hline $5 / 7 / 2009$ & 12 & & cuo & $\mathrm{Ti}$ & https://doi.org/10.1021/ja903726m \\
\hline $6 / 21 / 2004$ & & 8 & cub & $\mathrm{Zn}$ & https://doi.org/10.1021/ja905557z \\
\hline $8 / 27 / 2009$ & 12 & & - & $\mathrm{Be}$ & https://doi.org/10.1021/ja9072707 \\
\hline $4 / 6 / 2010$ & 12 & & cuo & $\mathrm{Ni}$ & https://doi.org/10.1021/ja102862j \\
\hline $11 / 4 / 2010$ & 6 & & oct & Co & https://doi.org/10.1002/chem.201003173 \\
\hline $1 / 4 / 2011$ & & 24 & rco & $\mathrm{Zn}$ & https://doi.org/10.1039/C1CC00047K \\
\hline $4 / 8 / 2011$ & & 36 & - & $\mathrm{Cu}$ & https://doi.org/10.1039/C1CC11996F \\
\hline $8 / 30 / 2011$ & 9 & & tct & $\mathrm{Ni}$ & https://doi.org/10.1038/ncomms1654 \\
\hline 10/6/2011 & & 12 & tte & $\mathrm{Zn}$ & https://doi.org/10.1038/ncomms1618 \\
\hline $10 / 3 / 2012$ & 6 & & oct & $\mathrm{Cu}$ & https://doi.org/10.1039/C2CE26611C \\
\hline $4 / 23 / 2012$ & 8 & & cub & $\mathrm{Zr}$ & https://doi.org/10.1021/ic300825s \\
\hline $7 / 6 / 2012$ & & 12 & cuo & $\mathrm{Cu}$ & https://doi.org/10.1039/C2CC34840C \\
\hline 2/7/2013 & 12 & & cuo & $\mathrm{RE}$ & https://doi.org/10.1021/ja401429x \\
\hline 6/7/2013 & 24 & & rco & $\mathrm{Zn}$ & https://doi.org/10.1038/srep02616 \\
\hline $8 / 5 / 2013$ & 6 & & $6 \mathrm{MR}$ & $\mathrm{Zr}$ & https://doi.org/10.1021/ja408084j \\
\hline $10 / 7 / 2013$ & 18 & 18 & eto & $\mathrm{RE} / \mathrm{Cu}$ & https://doi.org/10.1038/NCHEM.1982 \\
\hline $1 / 12 / 2014$ & 12 & & ico & $\mathrm{Zr}$ & https://doi.org/10.1021/ja500330a \\
\hline $1 / 12 / 2014$ & 10 & & - & $\mathrm{Zr}$ & https://doi.org/10.1021/ja500330a \\
\hline $1 / 12 / 2014$ & 6 & & oct & $\mathrm{Zr}$ & https://doi.org/10.1021/ja500330a \\
\hline $2 / 14 / 2014$ & 12 & & - & $\mathrm{Al}$ & https://doi.org/10.1021/ja501606h \\
\hline $10 / 17 / 2014$ & 12 & & D6R & $\mathrm{Zr}$ & https://doi.org/10.1021/ja510525s \\
\hline $1 / 12 / 2015$ & 8 & & cub & RE & https://doi.org/10.1021/jacs.5b00450 \\
\hline $1 / 12 / 2015$ & 12 & & D6R & $\mathrm{RE}$ & https://doi.org/10.1021/jacs.5b00450 \\
\hline $6 / 5 / 2015$ & 6 & & tpr & $\mathrm{Ti}$ & https://doi.org/10.1002/anie.201505512 \\
\hline $1 / 30 / 2016$ & & 6 & tpr & $\mathrm{Zn}$ & https://doi.org/10.1021/jacs.6b01093 \\
\hline $1 / 4 / 2017$ & 18 & & etg & $\mathrm{Hf}$ & https://doi.org/10.1021/jacs.7b00106 \\
\hline $5 / 5 / 2017$ & 6 & & oct & $\mathrm{RE}$ & https://doi.org/10.1021/jacs.7b04532 \\
\hline $8 / 15 / 2017$ & 4 & & $4 \mathrm{MR}$ & $\mathrm{Zr}$ & https://doi.org/10.1038/s41467-018-04152-5 \\
\hline $10 / 25 / 2017$ & 6 & & tpr & $\mathrm{Zr}$ & https://doi.org/10.1021/acsami.7b16163 \\
\hline $10 / 28 / 2017$ & 8 & & cub & $\mathrm{RE}$ & https://doi.org/10.1021/acsami.7b16380 \\
\hline $10 / 17 / 2017$ & 16 & & cuo & $\mathrm{Ti}-\mathrm{Zr}$ & https://doi.org/10.1021/acscentsci.8b00073 \\
\hline $2 / 15 / 2018$ & 8 & & cub & $\mathrm{Ti}-\mathrm{Ca}$ & https://doi.org/10.1002/anie.201802089 \\
\hline $3 / 2 / 2018$ & 6 & & $6 \mathrm{MR}$ & RE & https://doi.org/10.1002/anie.201802661 \\
\hline $3 / 10 / 2018$ & 9 & & cupola & $\mathrm{Zr}$ & https://doi.org/10.1021/acsami.8b03987 \\
\hline $1 / 4 / 2019$ & 9 & & cupola & RE & https://doi.org/10.1021/jacs.9b00122 \\
\hline 6/10/2019 & 12 & & $\mathrm{D} 6 \mathrm{R}$ & $\mathrm{Np}$ & https://doi.org/10.1021/jacs.9b06187 \\
\hline 6/30/2019 & 12 & & cuo & Co & https://doi.org/10.1039/c9sc03224j \\
\hline $10 / 19 / 2019$ & 6 & & oct & RE & https://doi.org/10.1021/jacs.9b11260 \\
\hline $1 / 17 / 2020$ & 12 & & twc & $\mathrm{Zr}$ & https://doi.org/10.1021/jacs.0c00637 \\
\hline $10 / 14 / 2020$ & 3 & & $3 M R$ & $\mathrm{Cu}$ & https://doi.org/10.1002/anie.202013839 \\
\hline $11 / 12 / 2020$ & 12 & & D6R & $\mathrm{Zr}$ & https://doi.org/10.1021/jacs.0c11881 \\
\hline
\end{tabular}

